Chubb, T.A. II. Inhibited Diffusion Driven Surface Transmutations. in Eleventh International Conference on

Condensed Matter Nuclear Science. 2004. Marseille, France.

\title{
II. Inhibited Diffusion Driven Surface Transmutations
}

\author{
Talbot A. Chubb \\ Greenwich Corp., 5023 N. 38th St., Arlington VA 22207, USA, tchubb@aol.com
}

\begin{abstract}
This paper is the second of a set of 3 papers dealing with the role of coherent partitioning as a common element in Low Energy Nuclear Reactions (LENR), by which is meant cold-fusion related processes. This paper discusses the first step in a sequence of 4 steps that seem to be necessary to explain Iwamura 2-alpha-addition surface transmutations. Three concepts are examined: saltmetal interface states, sequential tunneling that transitions $\mathrm{D}^{+}$ions from localized interstitial to Bloch form, and the general applicability of 2-dimensional vs. 3-dimensional symmetry hosting networks.
\end{abstract}

\section{INTRODUCTION}

Iwamura et al. ${ }^{1}$ have developed a LENR process that converts surface Cs atoms into surface $\operatorname{Pr}$ atoms using deuterium permeation through a specially structured, largely metal assembly. The assembly consists of a Pd substrate on which are deposited 4 layers of 2-nm $\mathrm{CaO}$ overcoated with 8$\mathrm{nm} \mathrm{Pd}$, plus $1 \mathrm{CaO}$ layer overcoated with a 40-nm Pd inflow layer. The Iwamura assembly is used as a permeable barrier between a $\mathrm{D}_{2}$-pressurized volume and a vacuum volume.

\section{ENVISIONED PROCESS}

I speculatively model the process as follows. At a $\mathrm{CaO}, \mathrm{Pd}$ interface, a diffusing deuteron is converted into a coherently-partitioned, nuclearly reactive Bloch form $\mathrm{D}^{+}$Bloch. ${ }^{2}$ Multiple Bloch deuterons organize themselves into a many-body Bloch-ion subsystem. Independent subsystems form on different $\mathrm{CaO}$ crystallites, as shown in Fig. 1. In these subsystems Bloch deuterons fuse, creating Bloch helium ions ${ }^{4} \mathrm{He}^{++}$Bloch. In a second step two ${ }^{4} \mathrm{He}^{++} \mathrm{Bloch}$ fuse, creating a product nucleus ${ }^{8} \mathrm{Be}^{4+} \mathrm{Bloch}$, which is stabilized by being coherently partitioned. The ${ }^{8} \mathrm{Be}^{4+} \mathrm{Bloch}^{4}$ energized by increases in coherent partitioning in a process that uses nuclear energy to mobilize the Be ion. The ${ }^{8} \mathrm{Be}^{4+}$ Bloch nucleus spreads out beyond the hosting $\mathrm{CaO}$-metal interface, spreading along accessible Pd-crystallite boundaries, including the interfaces between metal and gas, and metal and vacuum. A Cs atom protruding above the mean surface of the Pd metal is overlapped and infiltrated by the ${ }^{8} \mathrm{Be}^{4+}$ Bloch. The Cs nucleus combines with and absorbs the ${ }^{8} \mathrm{Be}^{4+} \mathrm{Bloch}$ nucleus, and becomes a Pr atom. Both the formation of Bloch helium and the transmutation of surface Cs into Pr are exothermic processes. 


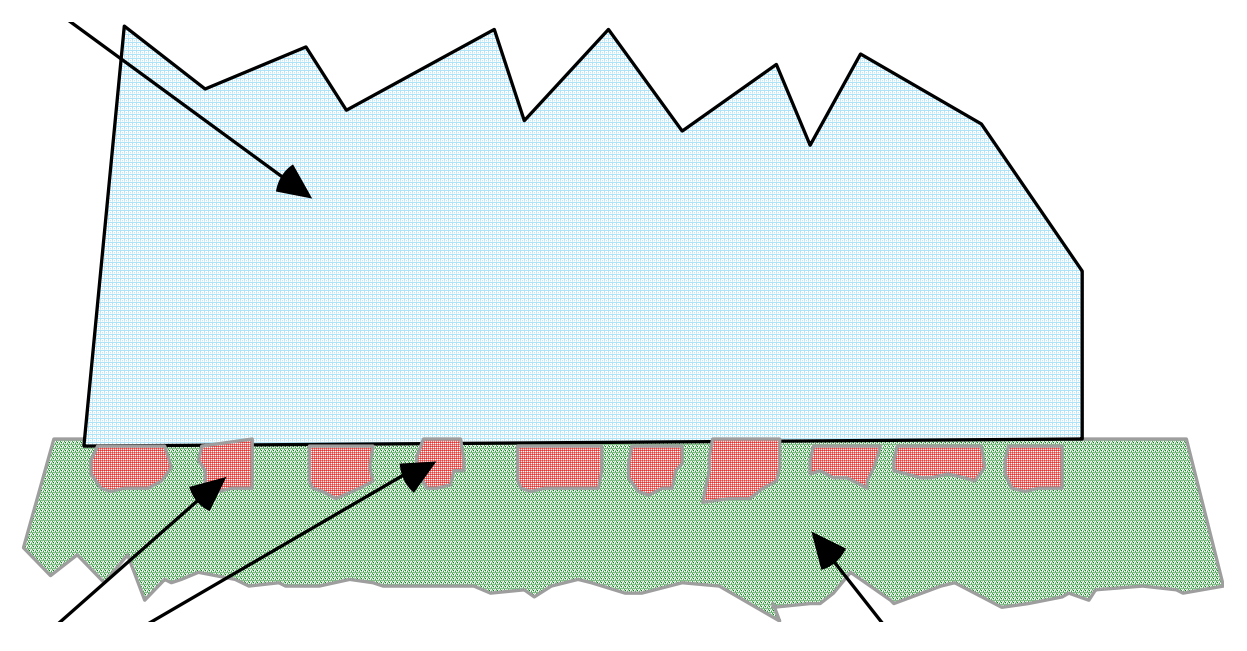

Fig. 1. Multi-crystallite modeling of Iwamura reactor plate, showing contacts between Pd substrate, sputtered $\mathrm{CaO}$ deposition layer, and sputtered $\mathrm{Pd}$ metal overcoat. The $\mathrm{CaO}$ is shown as a discontinuous set of ionic nano-crystals.

\section{PRIMARY CHALLENGE}

The core question is: Why does Bloch deuterium form within the $\mathrm{CaO}$,metal interfaces? My guess is that within the interface layer the Bloch deuterium configuration has lower free energy than such discrete particles as $\mathrm{D}^{+}, \mathrm{D}^{-}$, or adsorbed

\section{Salt-Metal-Interface Bloch Deuterium}

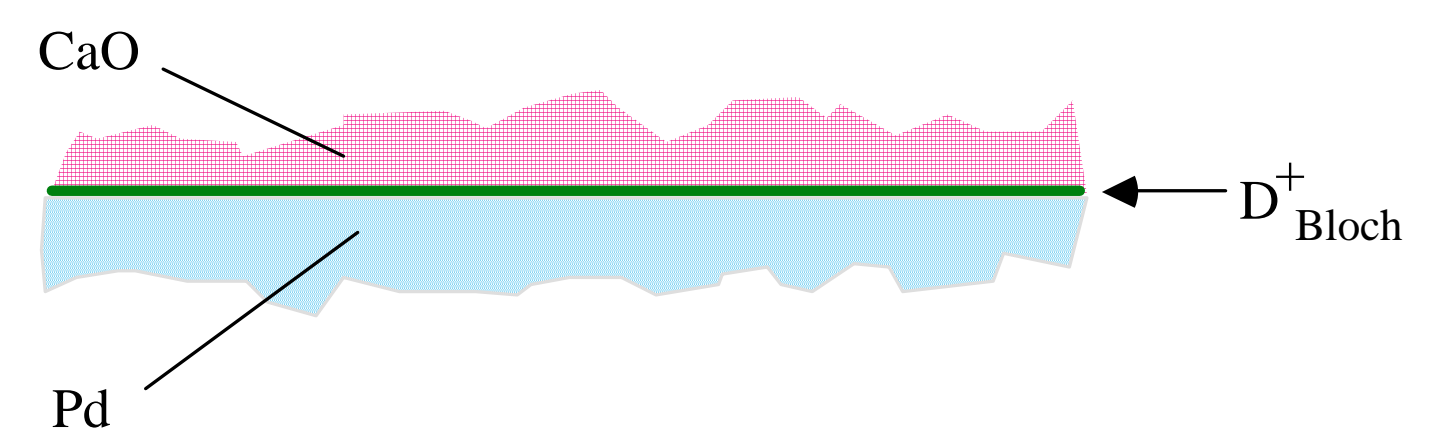

Fig. 2. Fine scale view of nano-portion of interface between ionic crystallite $\mathrm{CaO}$ and transition metal Pd, as fabricated for the Iwamura reactor. Arrow points to the location of the hypothesized $\mathrm{D}^{+}$Bloch deuteron. The $\mathrm{D}^{+}$Bloch ion is a nucleus with 2-dimensional symmetry matching the template provided by the $\mathrm{CaO}$ crystallite, and is neutralized (dressed) by the metal's electron Fermi sea. 
free-radical D atoms. This is generally not the case at the metal-gas and metal-vacuum interfaces, where electron-neutralized surface $\mathrm{D}^{+}$ions must be in excited states to be of Bloch configuration. ${ }^{3}$ Bulk $\mathrm{CaO}$ is a face centered $\mathrm{NaCl}$ lattice with a large Gibbs free energy of $-898 \mathrm{~kJ} / \mathrm{mol}$. One might imagine that a $\mathrm{CaO}$ crystal, with its alternating positive ion, negative ion structure, when interfaced with deuterided $\mathrm{Pd}$ could host a fraction of a $\mathrm{D}^{+} \mathrm{Bloch}$ ion adjacent to each interface $\mathrm{O}^{--}$ion and a fraction of $\mathrm{a} \mathrm{D}^{-}$Bloch ion adjacent to each interface $\mathrm{Ca}^{++}$ion. Each fractionally distributed Bloch deuteron and its fractionally distributed neutralizing electron charge would be in Bloch-function configuration. In this picture the $\mathrm{CaO}$ lattice determines the Bloch periodicity, while the interface boundary electrons of the more plastic metal adjust to ensure unit cell neutrality. The result is a partitioned $\mathrm{D}^{+}$ion with a local maximum in density within each of a large number of non-selftrapping potential wells ${ }^{4}$. The essential step that transitions localized $\mathrm{D}^{+}$into a delocalized Bloch configuration presumably occurs within these $\mathrm{CaO}-\mathrm{Pd}$ interface layers, as discussed later. Bloch deuteron pairs then convert into wavelike ${ }^{4} \mathrm{He}^{++} \mathrm{Bloch}$, which participate in Step 2 of the Iwamura process.

\section{IMPACT ON COLD FUSION}

The Iwamura et al. alpha-addition transmutation discoveries are the first of two new discoveries that clarify the role of coherent partitioning and Bloch-sensitivity in LENR processes. The Iwamura program started as a Mitsubishi Heavy Industries research study exploring anomalous FleischmannPons (F-P) emissions. Mitsubishi support allowed the science team to evolve their program into use of permeation as a means of avoiding electrolysis contamination worries. The Iwamura protocol is a non-electrolytic deuterium-palladium process by which surface Cs atoms have been repeatedly transmuted into surface Pr atoms. The Iwamura team has identified a new class of transmutations. The archetype is ${ }^{133} \mathrm{Cs}+2$ alpha $\rightarrow{ }^{141} \mathrm{Pr}$, which is a type of "alpha-addition transmutation". In the Iwamura process deuterium is forced to diffuse through Pd metal by exposing the front surface of the previously described special Pd plate reactor to $\mathrm{D}_{2}$ gas at 1 -atmosphere pressure at $70^{\circ} \mathrm{C}$, while the gas from the back side of the plate is pumped away using a vacuum pump. The transmutations do not take place without the use of a complex plate reactor. The specified design describes a Pd foil support plate of $0.1 \mathrm{~mm}$ thickness, on which are sputter-deposited a set of diffusion-inhibiting layers. Inside the plate there are five sputter-deposited 2-nm layers of $\mathrm{CaO}$ salt, separated from each other by sputter-deposited 8-nm layers of Pd metal, and topped with a 40-nm layer of sputter-deposited Pd. A sub-monolayer coating of Cs atoms is deposited on the top surface. The transmutation of Cs into Pr occurs during week-long permeation runs. Cs and Pr surface concentrations are measured by x-ray photoelectron spectroscopy (XPS) before and after permeation, and twice during imposed run interruptions. The salt layers, likely in the form of small $\mathrm{CaO}$ crystals, partially block the deuterium atoms as they work their way through the Pd plate. Transmutation is not observed if H-permeation is substituted for D-permeation, or if the $\mathrm{CaO}$ layers are not included in the plate structure.

The Iwamura process differs from F-P methods in that it avoids electrolysis. The F-P process uses overvoltage electrolysis to create a $\mathrm{PdD}_{\mathrm{X}}$ interstitial deuteride with $\mathrm{x} \equiv \mathrm{D} / \mathrm{Pd}>0.85$. To achieve $\mathrm{x}>0.85$ by equilibrium chemistry requires $\mathrm{D}_{2}$ pressures $>$ several kbar. ${ }^{5}$ On the other hand, both processes release nuclear energy from exothermic nuclear reactions, and transfer the released 
nuclear energy in a manner that heats a hosting metal lattice. Step 1 in the Iwamura process takes place at less than standard deuterium chemical potential.

The Iwamura process imposes challenges to cold fusion modelers. To model the Iwamura process one must explain: 1) why nuclear energy release occurs at relatively low $\mathrm{D}_{2}$ chemical potential, 2) how a process made possible by interior $\mathrm{CaO}$ crystals can effect transmutation on a distant metal surface, 3) how the transmutation of a high $\mathrm{Z}$ nucleus can occur, and 4) why the transmutation involves addition of two alphas instead of one. This paper is concerned mainly with Challenge 1. Challenges 2-4 are addressed in Paper III.

\section{LOW D ${ }^{+}$Bloch CONCENTRATION PROBLEM}

Let us consider Iwamura's success in achieving nuclear effects at relatively low $\mathrm{D}^{+} \mathrm{Bloch} / \mathrm{Pd}$ ratio. As described above, it is suggested that the periodic structure provided by the $\mathrm{CaO}$ salt crystallites helps create a one layer thick interface volume unusually suitable for hosting coherently partitioned Bloch deuterons. $\mathrm{CaO}$ is an ionic salt like $\mathrm{NaCl}$, and has a large negative Gibbs free energy, which suggests that its ordered form is not easily altered. In this speculation, the $\mathrm{CaO}$ forms the template for wave-like deuterium, and the metal's electrons forms the negative charge cloud that converts a positive ion into the neutral "atom" form.

\section{INITIAL STEP IN COHERENT PARTITIONING}

A relatively simple process can be envisioned by which the Iwamura plate reactor creates coherently partitioned deuterons. An incident $\mathrm{D}_{2}$ gas molecule strikes the Pd top surface and dissociates into two adsorbed D atoms. Each adsorbed D atom enters the Pd top surface by tunneling into an interior self-trapping octahedral site within the fcc lattice. The permeating deuteron then diffuses through the top sputtered Pd layer in response to an internal deuteron concentration gradient. Some of the deuterons encounter a $\mathrm{CaO}$ crystallite during the diffusion process. After hopping into one of the potential wells provided by the $\mathrm{CaO}$-metal interface volume, it tunnels through a low potential barrier to an adjacent potential well within the interface's 2dimensionally ordered periodic subsystem. Because of the low potential barrier between potential wells of the 2-dimensional symmetry interface sub-region, and because of the non-self-trapping character ${ }^{4}$ of the trapping sites, this tunneling splits the wave function into 2 coherent parts, as

described in Merzbacher's treatment of a particle in a "double-oscillator" potential. 6 The Merzbacher solution of the 2-potential well problem describes a single particle which is split between 2 potential wells. The split-particle wave function has the same wave function coherency that characterizes a single particle bound inside a single potential well. Thus the transition between single well geometry and double well geometry preserves wave-function phase order. Applied to the Iwamura geometry, it transiently creates a double-maximum coherent deuteron configuration. Further tunnelings further coherently delocalize the wave function, completing a transition from a double-maximum wave function into a coherently-partitioned $\mathrm{N}_{\text {well-maximum }}$ Bloch form.

\section{CAUTIONS}

It is probably a mistake to assume that cold fusion processes are always associated with 2dimensional symmetry Bloch deuterons. The heat release associated with Iwamura transmutations is in the low $\mathrm{mW}$ range, whereas a number of F-P related studies have resulted in heat release in the multiple Watt range. The Iwamura Cs studies do not include calorimetric measurement of heat; 
hence it is unknown to what extent additional cold fusion heat release occurs concurrently with transmutation heat generation. For F-P studies involving bulk Pd, it is the consensus view that measurable excess heat is normally produced in $\mathrm{PdD}_{\mathrm{X}}$ with $\mathrm{x}>0.85$. At $\mathrm{x}>0.7$, it becomes thermodynamically possible to begin to have some small fraction of the interstitial deuteron population occupying 3-dimensional-symmetry bulk-Pd tetrahedral sites. ${ }^{7}$ Some modelers suggest that tetrahedral site occupation by a fraction of the interstitial deuterons present in the bulk is required for F-P heat generation.

It is important to consider the work of Arata and Zhang (A-Z). A-Z have studied excess heat generation occurring in deuterided $\mathrm{Pd}$ in the form of fine powders, such as Pd-black. ${ }^{8}$ This powder has a nm-scale structure, as evidenced by a broadening of its $\mathrm{X}$-ray Bragg reflection peaks. A-Z have suggested that the powder may include domains having atom cluster order, as described by Fujita ${ }^{9}$. They have also suggested that high surface mobility is involved, and have used the term "spillover deuterium" to describe this property. "Spillover deuterium" is a term used in catalyst literature to describe anomalously high hydrogen activity.

Recently A-Z have explored use of powders produced by oxidizing amorphous Pd-Zr alloys. 10 These powders are very effective absorbers of deuterium gas. If all the absorbed gas is absorbed in the $\mathrm{Pd}$ fraction, which is not oxidized, the $\mathrm{D} / \mathrm{Pd}$ ratio approaches 3, i.e., the $\mathrm{PdD}_{\mathrm{X}} \rightarrow \mathrm{PdD} 3$. In a recent paper 11 not yet published in English, Arata has suggested that the structures produced by deuteriding these powders have fcc structures in which the octahedral sites are occupied by more than one deuteron. These expanded structures are presumably made possible by the large area/volume ratio of the powder, which reduces expansion stress relative to that of bulk metal. In his view, the potential well volumes surrounding the 2 tetrahedral sites and the incrementally available volume associated with a fully occupied octahedral site are about the same. He says that the tetrahedral sites can be transiently occupied. By the same argument the available volume for an incremental occupation of a fully occupied octahedral site can also be transiently occupied. In this view, the presence of these three transiently occupyable volumes in each unit cell forms an interconnecting network that runs through the metal atom cluster. It would constitute a 3dimensional symmetry network of low barrier potential wells. In such a network the "Merzbacher" coherent partitioning process could occur in the same manner as occurs within Iwamura's 2-dimensional symmetry CaO-Pd interface volume. Diffusing deuteron "visitors" would be converted into coherent Bloch form.

It is also important to note that an ordering of wave function phases should be able to occur without sequential tunneling in sufficiently low temperature Pd which has sufficient lattice array order. In the low temperature limit $\mathrm{T} \rightarrow 0$, spontaneous ordering should occur in fully loaded palladium $\mathrm{PdD}$. In $\mathrm{PdD}_{1+\delta} \delta$, the $\delta$-deuteron fraction should exist in a coherently partitioned Bloch form so as to avoid the free energy cost of breaking lattice symmetry. ${ }^{12}$ F-P cold fusion could then take place in a mode that does not break lattice symmetry in a Mossbauer-type momentumpreserving process. Reaction energy goes into the recoil energy of the coherently partitioned Bloch product ${ }^{4} \mathrm{He}^{++}$Bloch and the recoiling crystallite. This type of fusion does not require the irreversibility step associated with electron scattering. For example, a recoiling host occurs during the Mossbauer energy release from an iron-containing lattice in a process that balances the 
momentum of a released gamma ray. The de-excited Fe atom recoils as part of the recoiling lattice crystallite. The spontaneous ordering would be an example of the ordering that occurs in many-

body lattice systems. 13 However, my present view is that it is unlikely that spontaneous ordering is occurring in the above-room-temperature environment that characterizes the Iwamura process.

\section{REFERENCES}

1. Iwamura, Y., M. Sakano, and T. Itoh, "Elemental Analysis of Pd Complexes: Effects of D2 Gas Permeation", Jpn. J. Appl. Phys. 41, 4642 (2002).

2. Chubb, T. A., "The dd Cold Fusion-Transmutation Connection", Proc. ICCF10, (2004). Chubb, T. A., "Bloch Ions", This Proceedings.

3. Puska, M. J. and R. M. Nieminen, "Hydrogen Chemisorbed on Nickel Surfaces: A WaveMechanical Treatment of Proton Motion", Surface Science 157, 413 (1985). See Fig. 13.

4. Chubb, T. A., "LENR: Superfluids, Self-Trapping and Non-Self-Trapping States", Proc. ICCF10, (2004) in Press.

5. Baranowski, J. "Metal Hydrogen Systems at High Hydrogen Pressure", Hydrogen in Metals II, ed. Alefield and Volkl, (Springer-Verlag, Berlin, 1978), p. 157. Fig. 4.5 shows pressure for $\mathrm{H} / \mathrm{Pd}$. Pressures for D/Pd are higher. Taken from lecture by S. Crouch-Baker.

6. Merzbacher, E., "Quantum Mechanics", (John Wiley \& Sons, New York, 1964) pp. 64-77.

7. Oriani, R. A., "The Physical and Metallurgical Aspects of Hydrogen in Metals", Trans. Fusion Technol. 47, ICCF4, 235 (1994). See p. 239.

8. Arata, Y. and Y-C Zhang, "A New Energy Caused by 'Spillover Deuterium'", Proc. Japan Acad. 70B, 106 (1994).

9. Fujita, H., "Studies on Atom Clusters by Ultra-High Voltage Electron Microscopy", Mater. Trans., JIM, 35, 563-575, (1994).

10. Arata, Y. and Y-C Zhang, "Development of Compact Nuclear Reactor Using Solid Pycnodeuterium as Nuclear Fuel", Proc. ICCF10, (2004) in Press..

11. Arata, Y. . "The Formation of 'Solid Deuteriums" Solidified inside Crystal Lattice and Intense Solid State Nuclear Fusion, translation to be published in 21st CenturyScience, (2005)

12. Chubb, T. A. and S. R. Chubb, "Cold Fusion as an Interaction between Ion Band States", Fusion Technol. 30, 93 (1991).

13. Chubb, S. R. and T. A. Chubb,"Relationship Between Microscopic and Macroscopic Interactions in Low Energy Nuclear Reactions", Proc. ICCF9, Edited by Xing Z. Li, (Tsinghua University Press, Beijing, 2003) 57. 